\title{
Investigation of $1 D$ Siliver Nanoparticle Arrays for Use as Molecule Concentration-Specific SERS Substrates
}

\author{
Jun Gao, ${ }^{1,2}$ Huan Qian, ${ }^{2}$ Shang $X{ }^{2}{ }^{2}$ and Min $\operatorname{Han}^{2}$ \\ ${ }^{1}$ College of Physics \& Electronic Engineering, Changshu Institute of Technology, Changshu 215500, China \\ ${ }^{2}$ National Laboratory of Solid State Microstructures and Department of Materials Science and Engineering, Nanjing University, \\ Nanjing 210093, China
}

Correspondence should be addressed to Min Han; sjhanmin@nju.edu.cn

Received 1 June 2013; Revised 14 November 2013; Accepted 15 November 2013

Academic Editor: Raymond Whitby

Copyright (C) 2013 Jun Gao et al. This is an open access article distributed under the Creative Commons Attribution License, which permits unrestricted use, distribution, and reproduction in any medium, provided the original work is properly cited.

\begin{abstract}
One-dimensional arrays of silver nanoparticles with a particle size of $10.5 \mathrm{~nm}$ and interparticle spacing of $8.5 \mathrm{~nm}$ were fabricated by depositing nanoparticles in gas phase on block copolymer self-assembled templates. The substrate showed a surface-enhanced Raman scattering (SERS) enhancement factor as high as $1 \times 10^{7}$ with good reproducibility and stability. The dependence of the average enhancement factor and the SERS intensity on the packing density of the analyte molecules were investigated. For a tiny amount of analytes in the range of $1 \times 10^{-14}$ to $3 \times 10^{-13} \mathrm{~mol} / \mathrm{mm}^{2}$, the SERS signal showed a linear dependence on the molecule packing density on a logarithmic scale, with a slope of about 1.25 . The substrates are promising for quantitative detection of trace level molecules.
\end{abstract}

\section{Introduction}

The last three decades have witnessed an ever-increasing interest in surface-plasmon-based analytical techniques, of which surface-enhanced Raman scattering (SERS) [1-5] is one of the most important applications. However, the rational design and facile fabrication of reproducible SERS substrates with large and stable enhancement of Raman signals remain a critical challenge $[6,7]$. Despite that extensive efforts have been devoted to the local field enhancement and single molecule detection at the hot spots, less attention has been paid to the average enhancement factor $\bar{G}$, which has greater relevance for the design and optimization of SERSbased chemical sensors $[8,9]$. Metal nanoparticle assemblies are important substrates for SERS-based molecule sensing, because of the light confinement between nanoparticles, which provides enhanced local electromagnetic fields and the tunability of localized surface plasmon resonances. To enable molecule detection in high SERS activity, stability and reproducibility, a knowledge of the arrangement of the analyte molecules and hot spots, and their effects to the average enhancement are important. To obtain a large average enhancement, it is crucial to provide a state of aggregation of the metal nanoparticles and to place the analyte molecules in the sites of the junctions of metal nanoparticles. In this sense, one- and two-dimensional ordered arrays of closely spaced metal nanoparticles can be excellent candidates for SERS substrates. They can provide not only giant field enhancement both through long range photonic coupling and the local electromagnetic near field confinement, but also high reproducibility because of the uniform and dense distribution of hot spots.

Recently, block copolymer self-assembled nanopatterns were used as templates to fabricate high density regular arrays of metal nanoparticles [10-12]. Excellent structure uniformity in the interparticle gap distance and the size of the nanoparticles in the array could be obtained in a large area, and high SERS enhancement factor with good reproducibility was demonstrated [13-18]. Furthermore, the morphology of the nanoparticle arrays can be adjusted by tailoring the block copolymer self-assembling to maximize the Raman scattering.

In this paper, densely packed one-dimensional arrays of silver nanoparticles were fabricated on large scale by depositing gas-phase generated nanoparticles with controlled size on block copolymer self-assembled templates. The 


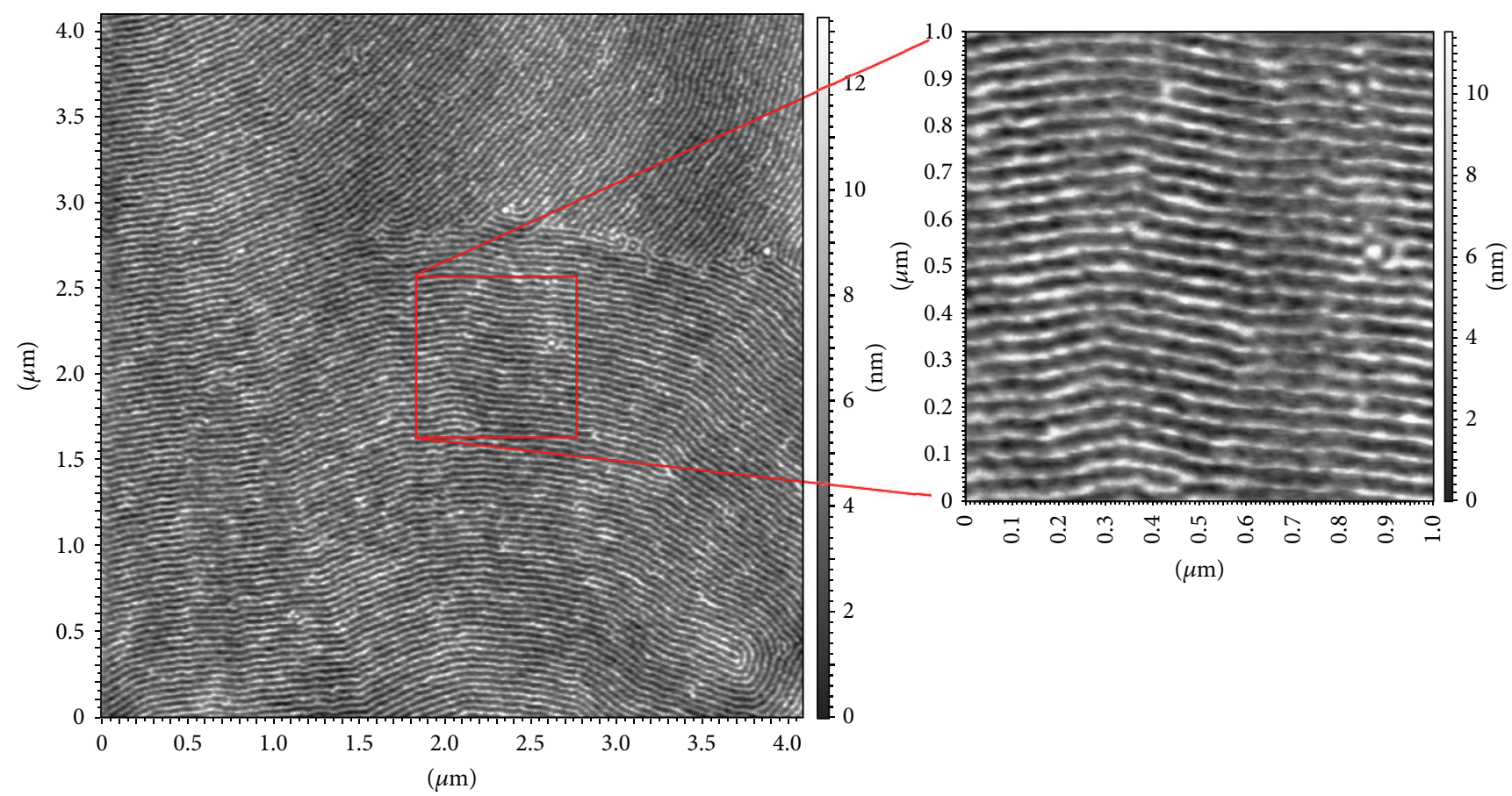

FIgURE 1: Typical $4 \times 4 \mu \mathrm{m}^{2}$ tapping-mode AFM height image of prepatterned SBS block copolymer films.

nanoparticle arrays were used as stable SERS substrates, and the dependence of the average enhancement factor on the packing density of the analyte molecules on the substrate was investigated. Specifically, for a tiny volume of very low concentration analytes that can only form a submonolayer on the substrate surface, a stable $\bar{G}$ factor as high as $10^{7}$ could be maintained, and the SERS signal of the analytes shows a near-unity power-law dependence on the molecule density. It therefore shows the possibility to be used for quantitative trace level detection and rapid analysis of molecules.

\section{Experimental Methods}

Triblock copolymer poly(styrene- $b$-butadiene- $b$-styrene) (SBS) films with self-assembled nanopatterns were used as templates to selectively adsorb the deposited silver nanoparticles on the polybutadiene (PB) phase $[11,19]$. The films were prepared by spin coating toluene solutions of $4 \mathrm{wt} \%$ SBS (weight-averaged molecular weight $\mathrm{M}_{w}=140000 \mathrm{Da}$, polydispersity index PDI $=1.2$, polystyrene (PS) weight fraction $f_{\mathrm{PS}}=0.3$, Aldrich Chemical Inc.) onto fused quartz glasses at about $2500 \mathrm{rpm}$ for $30 \mathrm{~s}$, followed by solvent annealing in a toluene vapor atmosphere with an extremely slow toluene evaporation rate $(\sim 0.001 \mathrm{~mL} / \mathrm{h})$ at room temperature. The solvent annealing went on for 3 days, after which the morphologies of the block copolymer films were characterized in air with an atomic force microscope (AFM, NTEGRA Probe NanoLaboratory, NT-MDT, Co.) working in tapping mode.

Silver nanoparticles were subsequently deposited onto the prepatterned SBS copolymer film surface under high vacuum at room temperature. The nanoparticles were generated by a magnetron plasma gas-aggregation cluster source $[20,21] .200 \mathrm{sccm}$ of argon were introduced into the liquid nitrogen cooled aggregation tube to maintain a stable gas flow with a pressure of $180 \mathrm{~Pa}$. A stable magnetron discharge was operated with an input power of $90 \mathrm{~W}$. Atoms were sputtered from the silver target and nanoparticles were formed through the aggregation process in the argon gas. The nanoparticles were extracted to the high vacuum deposition chamber through a differential pumping system. The substrate was placed in the deposition chamber perpendicular to the nanoparticle beam. Preformed nanoparticles were deposited at low kinetic energy $(<0.1 \mathrm{eV} /$ atom $)$ onto the substrate surfaces. The deposition rate was monitored by a quartz crystal microbalance and controlled to be about $2 \AA /$ s. The coverage was controlled to be less than one monolayer by controlling the deposition time. The Ag nanoparticle covered SBS copolymer films were characterized with a transmission electron microscope (TEM).

Raman spectra were acquired on a confocal Raman spectroscope (NT-MDT NTEGRA Spectra) using a $473 \mathrm{~nm}$ laser excitation and a $70 \mu \mathrm{m}$ diameter pinhole. The laser beam was focused with an objective $(100 \times, \mathrm{NA}=0.9)$ to give an illumination area in $350 \mathrm{~nm}$ diameter on the sample surface. A constant laser power of $100 \mu \mathrm{W}$ was used for the SERS measurement.

\section{Results and Discussion}

Figure 1 shows a typical tapping-mode AFM height image of an SBS copolymer thin film. The film is composed of microphase-separated domains with cylindrical morphology 


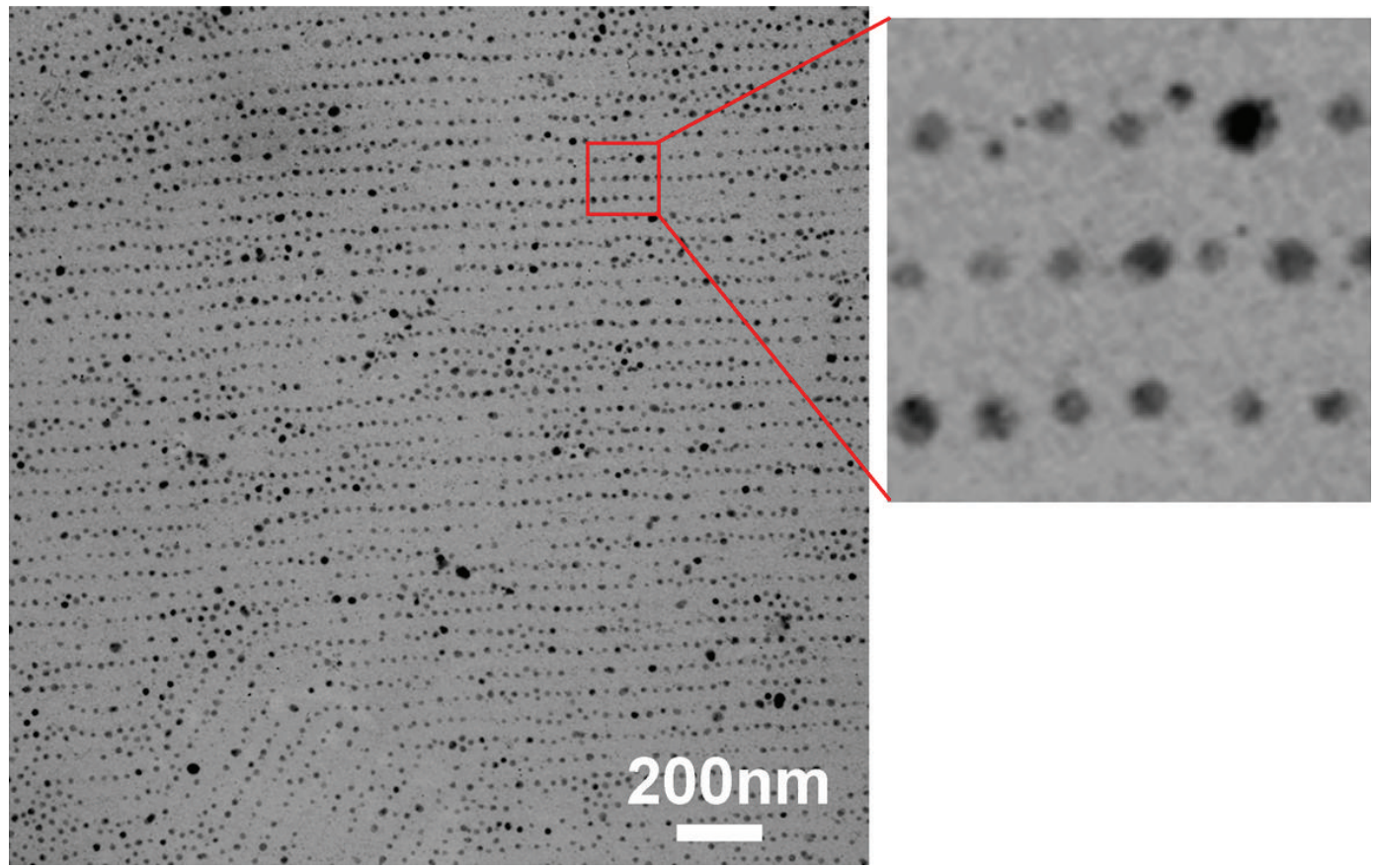

(a)

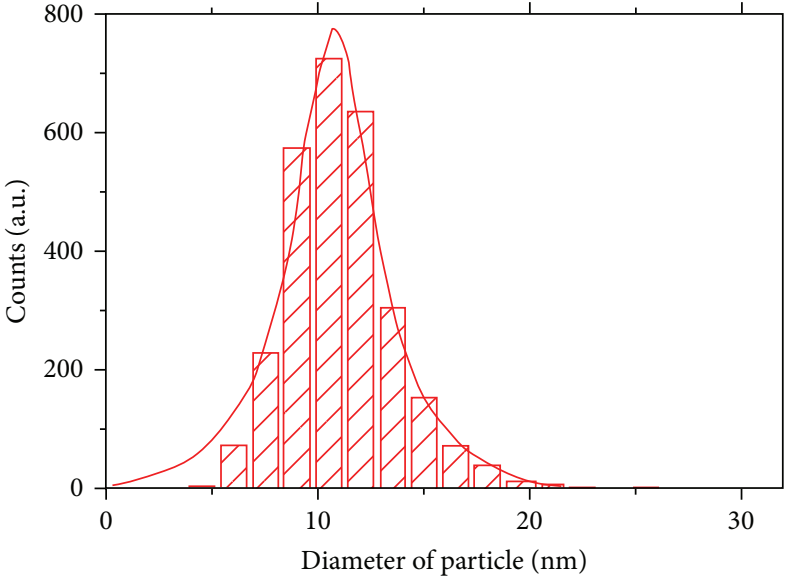

(b)

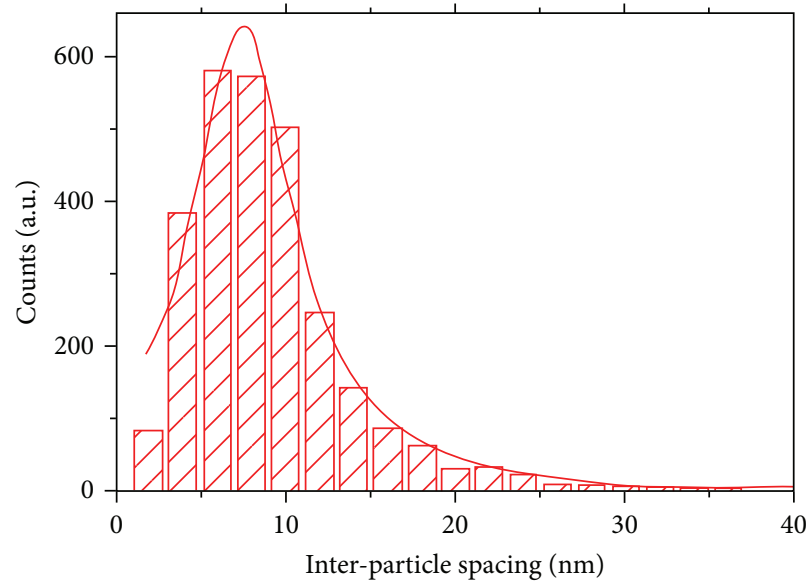

(c)

FIGURE 2: (a) A typical TEM image of the one-dimensional silver particle arrays on SBS prepatterned template. (b) The size distribution of the silver nanoparticle arrays. (c) The distribution of the interparticle spacings of the silver nanoparticle arrays.

aligned parallel to the substrate, similar to those found in our previous investigations [11]. The orientational order of the cylinders can cover several tens of lattice constant, which is around $38 \mathrm{~nm}$ as measured from the AFM image.

The Ag nanoparticle covered SBS copolymer films were characterized with a transmission electron microscope (TEM). As shown in Figure 2(a), the silver nanoparticles assemble to chain-like arrays with nearly equal interchain spaces, approximately $38 \mathrm{~nm}$, which corresponds to the period of the in-plane PS domain cylinders in the underlying SBS copolymer substrate. It has been demonstrated previously that evaporating metal onto a phase-separated block copolymer film resulted in selective decoration of one of the blocks $[10,22]$. When silver nanoparticles are deposited onto the triblock copolymer SBS thin films, they are selectively absorbed onto the $\mathrm{PB}$ domains, forming regular arrays of nanoparticles [11]. Due to the high stability of the nanoparticles preformed in gas phase and the limited diffusive mobility and coalescence of silver nanoparticles on the PB blocks, nanochains of particles with fairly narrow size distribution and uniform interparticle spacing are produced. With a carefully controlled coverage of silver nanoparticles, the SBS copolymer surfaces are uniformly distributed with nanoparticle chains. A single chain typically extends more than 1 micron, containing several tens of nanoparticles, covering the whole range of the phase separated pattern area with minor defect clusters. Figures 2(b) and 2(c) give the distributions of $\mathrm{Ag}$ nanoparticle size and the interparticle 


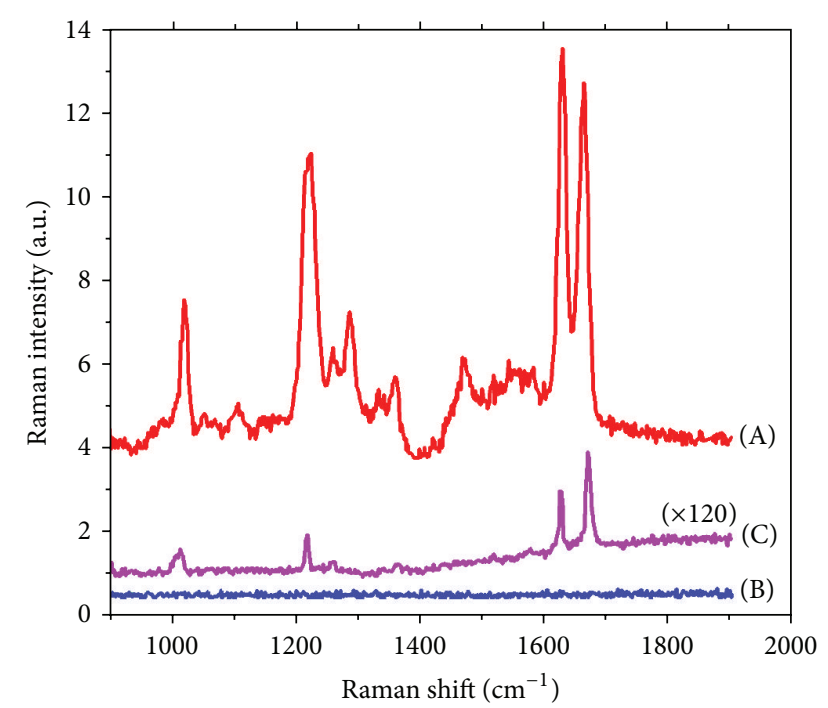

FIGURE 3: Raman spectra of BPE molecules adsorbed onto two different substrates. (A) $2 \times 10^{-6} \mathrm{M}$ of BPE solution drop casted on the SBS template surface uniformly distributed with Ag nanoparticle chains. (B) $2 \times 10^{-6} \mathrm{M}$ of BPE solution drop casted on pure silica glass surface. For spectrum (A) and (B), $100 \mu \mathrm{W}$ laser power was used. (C) $2 \times 10^{-3} \mathrm{M}$ of BPE solution drop casted on pure silica glass, $1 \mathrm{~mW}$ laser power was used. Spectrum (C) was magnified 120 times for clarity.

spacings within the chains. The nanoparticles have a mean diameter of about $10.5 \mathrm{~nm}$ and a standard deviation of about $2.5 \mathrm{~nm}$. The interparticle spacing within the chain is about $8.5 \mathrm{~nm}$ with a standard deviation of about $6.0 \mathrm{~nm}$. From Figure 2(a), the number density of the nanoparticles is measured to be about $2 \times 10^{3} \mu \mathrm{m}^{-2}$, which corresponds to that estimated according to the morphology of the nanoparticle arrays.

The SERS activity of the silver nanoparticle arrays on SBS templates was examined with trans-1,2-bi-(4-pyridyl) ethylene (BPE) molecules. $10 \mu \mathrm{L}$ of low concentration BPE methanol solution $\left(2 \times 10^{-6} \mathrm{M}\right)$ was dropped onto the substrates, with a surface area of $1 \mathrm{~cm}^{2}$. The liquid drop spread on the substrate surface and fast dried in air. Raman spectra measured from the substrates covered with or without Ag nanoparticle are plotted in Figure 3. Strong Raman bands can be observed (curve (A)) when the BPE molecules are attached to the silver nanoparticle covered substrates, while no Raman signal is detectable under the similar laser illumination condition if only pure BPE specimen is used (curve (B)). Obviously, Raman scattering is significantly enhanced by the silver nanoparticle arrays. The major peaks of the SERS spectra reveal the characteristic Raman bands of BPE, that is, $1640 \mathrm{~cm}^{-1}$ and $1610 \mathrm{~cm}^{-1}$ due to $(\mathrm{C}-\mathrm{C})$ stretching modes, $1200 \mathrm{~cm}^{-1}$ due to both stretching and rocking modes of $\left(\mathrm{C}_{r}-\right.$ $\mathrm{C}_{b}$ ) where $\mathrm{C}_{r}$ is a ring carbon attached to a bridging carbon and $\mathrm{C}_{b}$ is a bridging carbon, and $1010 \mathrm{~cm}^{-1}$ due to both $(\mathrm{C}-$ $\mathrm{C})$ and $(\mathrm{C}-\mathrm{N})$ stretching modes [23]. Between the major peaks, there are a number of minor peaks, contributed both from the Raman bands of BPE and SBS. With a moderate laser power, the spectral noise coming from Raman scattering of SBS substrate is not significant, even with an analyte concentration as low as $2 \times 10^{-6} \mathrm{M}$. This may be ascribed to the lower efficiency on the excitation and detection of the local field enhanced Raman scattering from SBS molecules, since the surface of the SBS substrate is covered by Ag nanoparticles.

The BPE Raman bands at 1610 and $1640 \mathrm{~cm}^{-1}$ were selected for evaluating the Raman enhancement factor. The average surface enhancement factor $\bar{G}$ is defined as

$$
\bar{G}=\left(\frac{I_{\text {SERS }}}{I_{\text {Raman }}}\right)\left(\frac{N_{\text {bulk }}}{N_{\text {ads }}}\right),
$$

where $I_{\text {SERS }}$ and $I_{\text {Raman }}$ are the integrated Raman intensities of BPE on SERS substrate and normal substrate, respectively. $N_{\text {bulk }}$ and $N_{\text {ads }}$ are the number of BPE molecules on normal substrate and SERS substrate, respectively. Since in the measurement of Raman spectroscopy for analytes on normal substrate, the laser power was 10 times larger than that used for SERS measurement, $I_{\text {Raman }}$ was normalized with respect to the laser power. From the Raman spectra shown in Figure 3, a surface enhancement factor of about $1.1 \times 10^{7}$ can be estimated.

We investigated the molecule concentration-specific SERS property of the one-dimensional Ag nanoparticle arrays. BPE was dissolved in methanol to form diluted solutions with a series of concentrations $\left(2 \times 10^{-4} \mathrm{M}, 7 \times\right.$ $10^{-5} \mathrm{M}, 2 \times 10^{-5} \mathrm{M}, 8 \times 10^{-6} \mathrm{M}, 3 \times 10^{-6} \mathrm{M}, 2 \times 10^{-6} \mathrm{M}, 1 \times$ $10^{-6} \mathrm{M}, 5 \times 10^{-7} \mathrm{M}$, and $1 \times 10^{-7} \mathrm{M}$, resp.). A series of surface densities of BPE molecules ranging from $2 \times 10^{-11} \mathrm{~mol} / \mathrm{mm}^{2}$ to $1 \times 10^{-14} \mathrm{~mol} / \mathrm{mm}^{2}$ were obtained corresponding to the solution concentrations, assuming that the molecules are uniformly distributed on the substrate free of aggregation. Their SERS spectra are plotted in Figure 4. For most of the concentrations, the major Raman bands of BPE, that is, 1640, 1610,1200 , and $1010 \mathrm{~cm}^{-1}$, are clearly visible. Only for the lowest concentration, $1 \times 10^{-7} \mathrm{M}$ which gives a corresponding molecular surface density of $1 \times 10^{-14} \mathrm{~mol} / \mathrm{mm}^{2}$, the characteristic Raman bands of BPE are almost submersed in the background level, indicating the detection limit of BPE is reached for the present SERS measurement configuration. For this concentration, the number of BPE molecules covered by the laser spot, with a diameter of about $350 \mathrm{~nm}$, is estimated to be about $6 \times 10^{2}$. With a rough estimation, these molecules cover less than $0.2 \%$ of the substrate surface, assuming that the area of a single BPE molecule is approximately $30 \AA^{2}$ [24]. Above this lowest concentration, reproducibility of SERS signals is quite well. The intensity of SERS signals acquired from various locations on the substrate show consistence within $18 \%$. To examine the uniformity of SERS signals across a large surface area, a micro-Raman map of sample area with $200 \mathrm{~nm}$ spot spacing was measured. Figure 5 shows a 3 -dimensional mapping of the integrated Raman intensity of the $1610 \mathrm{~cm}^{-1}$ and $1640 \mathrm{~cm}^{-1}$ band of BPE. Overall the Raman intensity is very uniform across the surface, indicating that both the distribution of the analyte molecules and the enhancement are uniform.

Figure 6(a) shows the integrated intensities of BPE Raman peaks at 1610 and $1640 \mathrm{~cm}^{-1}$ for various BPE molecule 


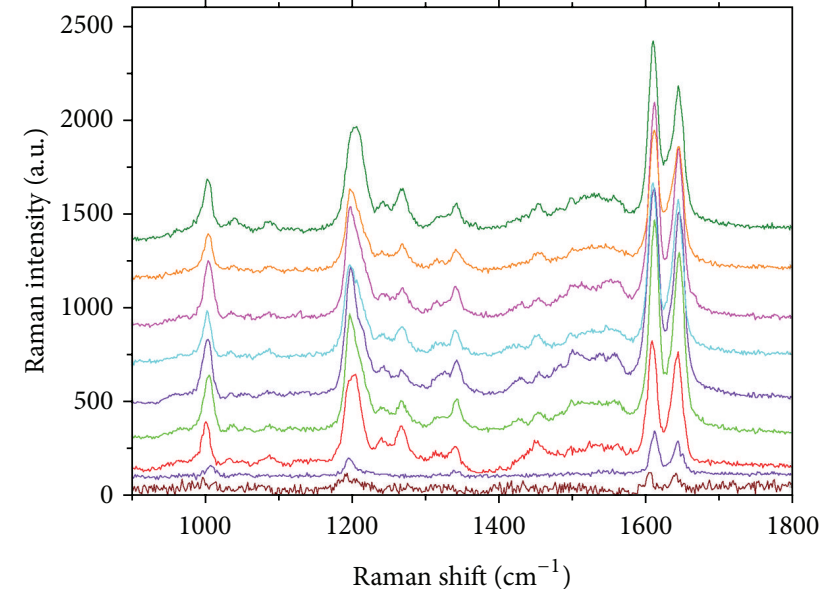

FIGURE 4: The representative surface enhanced Raman spectra of BPE molecules absorbed on silver nanoparticle arrays, with different concentrations. From upper line to bottom line, the corresponding BPE molecular surface packing densities are $2 \times 10^{-11} \mathrm{~mol} / \mathrm{mm}^{2}$, $7 \times 10^{-12} \mathrm{~mol} / \mathrm{mm}^{2}, 2 \times 10^{-12} \mathrm{~mol} / \mathrm{mm}^{2}, 8 \times 10^{-13} \mathrm{~mol} / \mathrm{mm}^{2}$, $3 \times 10^{-13} \mathrm{~mol} / \mathrm{mm}^{2}, 2 \times 10^{-13} \mathrm{~mol} / \mathrm{mm}^{2}, 1 \times 10^{-13} \mathrm{~mol} / \mathrm{mm}^{2}, 5 \times$ $10^{-14} \mathrm{~mol} / \mathrm{mm}^{2}$, and $1 \times 10^{-14} \mathrm{~mol} / \mathrm{mm}^{2}$. All spectra were recorded under identical experimental conditions and were vertically shifted for clarity.

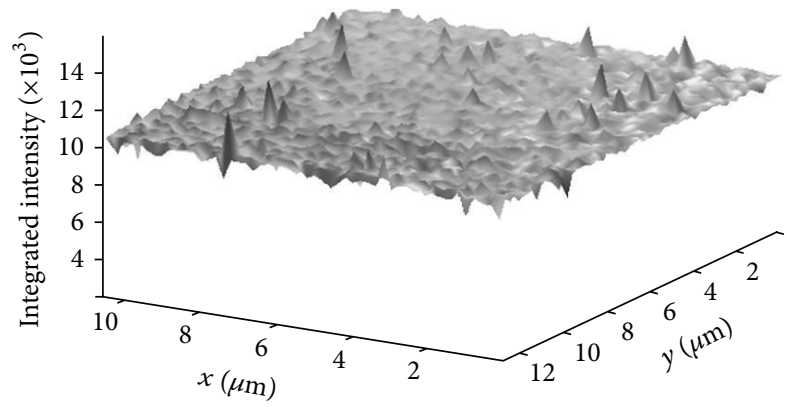

FIGURE 5: Micrograph of Raman mapping of the integrated intensity of the $1610 \mathrm{~cm}^{-1}$ and $1640 \mathrm{~cm}^{-1}$ Raman bands. BPE molecule density: $1 \times 10^{-13} \mathrm{~mol} / \mathrm{mm}^{2}$. Scanning step: $200 \mathrm{~nm}$.

densities. The standard deviations are obtained by averaging the SERS spectra of 20 individual spots all around the substrate. At higher BPE surface densities, from $3 \times$ $10^{-13} \mathrm{~mol} / \mathrm{mm}^{2}$ to $2 \times 10^{-11} \mathrm{~mol} / \mathrm{mm}^{2}$, the SERS intensity remains nearly unchanged. On the other hand, when the surface density of the BPE molecules is reduced to $3 \times$ $10^{-13} \mathrm{~mol} / \mathrm{mm}^{2}$, the SERS intensity decreases as the BPE surface density reduces, showing a linear dependence in the double-log plot, that is, a power law of the molecule packing density dependence, with a near unity exponent of about 1.25. This power law relation demonstrates that the SERS signals become more sensitive to the molecule surface density at such low packing densities, from $3 \times 10^{-13}$ to $1 \times 10^{-14} \mathrm{~mol} / \mathrm{mm}^{2}$, with a molecular coverage from $0.2 \%$ to $6 \%$. It means quantitative analysis of such low concentration molecules can be achieved through SERS measurement on

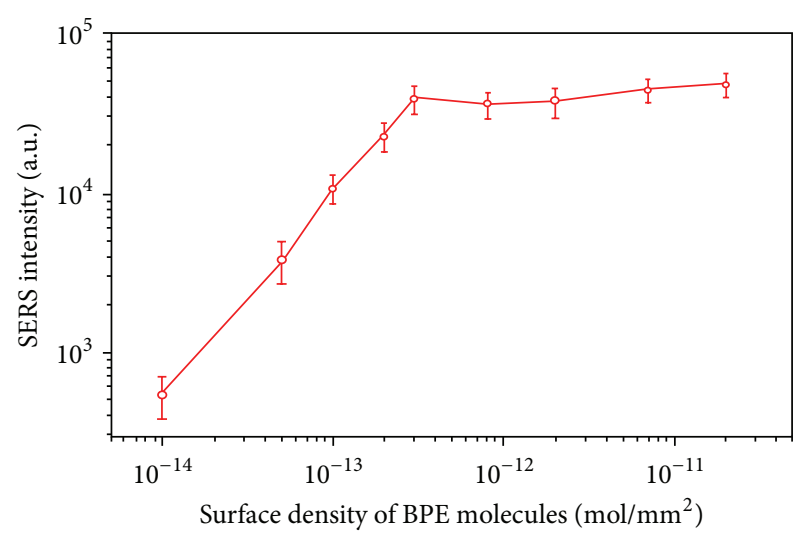

(a)

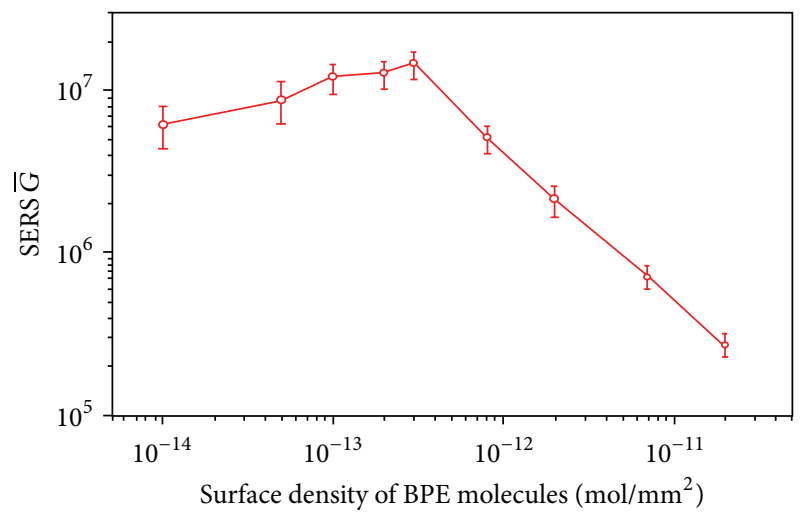

(b)

FIGURE 6: (a) SERS intensity and (b) SERS enhancement factor as a function of the surface packing density of BPE molecules calculated by the integrated area of BPE Raman bands at about $1610 \mathrm{~cm}^{-1}$ and $1640 \mathrm{~cm}^{-1}$.

the silver nanoparticle array substrates provided that the stability and reproducibility of these SERS substrates are obtained.

In order to take quantitative analysis of relation between SERS intensity and the molecular concentration, we consider the average Raman enhancement factor $\bar{G}$ at various BPE concentrations. It is clearly seen in Figure 6(b) that the average enhancement factor intensively changes with molecule packing density on the substrate at high molecular concentration. With the decrease of BPE surface density, the average Raman enhancement factor $\bar{G}$ increases and reaches a maximum, as large as $1.5 \times 10^{7}$, for the packing density of $3 \times 10^{-13} \mathrm{~mol} / \mathrm{mm}^{2}$, and then a stable value of $\bar{G}$ is maintained at about $1 \times 10^{7}$, and only a slight change takes place while the molecule packing density reduces by a factor of 30 , from $3 \times 10^{-13}$ to $1 \times 10^{-14} \mathrm{~mol} / \mathrm{mm}^{2}$. With such a stable average enhancement factor, a quantitative relationship arises between Raman signal intensities and molecular surface densities, as shown in Figure 6(a).

For the silver nanoparticle array SERS substrate, there are two kinds of SERS active points: the surface of metal nanoparticles and interparticle spacing (nanoparticle junction, or the so-called "hot spot"). The local electromagnetic 
field at the nanoparticle junction is much greater than that on the nanoparticle surface. Only Raman scattering from the molecules locating around the nanoparticle junctions can be enhanced greatly, whereas molecules out of the junction regions have much less microscopic enhancement. Therefore, a SERS signal results mostly from molecules located at the nanoparticle junctions, which may only account for a small portion of the molecules that distribute on the substrate or absorbed on the nanoparticle surfaces. The distribution of SERS active points with different microscopic enhancements and the real number of the analyte molecules that occupy the specific SERS active points have an especially strong influence on the average enhancement factor. At high molecule density, the available high field volumes at the nanoparticle junctions have fully been occupied, restricting the further access of analytes. In this sense, an increase of molecule density does not always cause a proportional increase of the number of molecules that contribute significantly to the SERS signal. Therefore, when the BPE molecule packing density is higher than $3 \times 10^{-13} \mathrm{~mol} / \mathrm{mm}^{2}$, only small changes on the SERS intensity can be observed as BPE molecule density increases, as shown in Figure 6(a), and accompany with an intensive reduction of the average Raman enhancement factor $\bar{G}$, as shown in Figure 6(b). On the contrary, at lower analyte concentration, adequate high field volumes at the nanoparticle junctions remain available for the access of analytes. With the increase of the BPE molecule density, more molecules access the high-field regions and contribute significantly to the SERS signals. Accordingly, the SERS intensity increases with the concentration of the analytes, whereas the average Raman enhancement factors $\bar{G}$ only shows a slight change. Since the number of molecules located in the hot spots increases proportionally to the total amount of analytes exposed to the probing laser, a constant specific SERS intensity normalized to the molecular concentration is achieved. A highest average Raman enhancement arises at the analyte concentration with which the volumes of the hot spots are almost fully occupied. There is a balance among the packing density of the molecules, the density, and uniformity of the SERS active points, as well as the volumes of the SERS active points that the analytes can access.

This mechanism leads to the strong sensitivity of SERS intensities to the arrangement of metal nanoparticles as well as the packing of the analyte molecules. Furthermore, the distribution uniformity of the hot spots not only affects the SERS activity, but also determines its stability and reproducibility. For the one-dimensional silver nanoparticle array we prepared, the well-controlled patterning geometry that enables a sensitive and monotonous dependence between SERS intensity and the molecular packing density ranging from $1 \times 10^{-14} \mathrm{~mol} / \mathrm{mm}^{2}$ to about $3 \times 10^{-13} \mathrm{~mol} / \mathrm{mm}^{2}$, covering about one and half order of magnitude, due to optimizing the high-field site distribution and the available high field volume. A wide range of analyte concentrations that have a monotonously dependent SERS intensity is important to a SERS substrate for trace level molecule detection.

The well-controlled patterning geometry is not only important to get high stability and reproducibility on SERS sensoring by providing uniform SERS active point distributions, but also important to get a large local field enhancement. The local electromagnetic fields can be largely enhanced due to the strong coherent coupling between nanoparticles in one-dimensional arrays [25]. An interparticle spacing of about $8.5 \mathrm{~nm}$ is measured for the onedimensional silver nanoparticle array we prepared. A red shift of the plasmon resonance wavelength was observed on such nanoparticle arrays [16], which could also contribute to the increase of local field enhancement by tuning the resonant excitation condition of the substrate to matching the excitation laser wavelength. Recently Mirkin and coworkers $[26,27]$ found that there is a nonzero optimum interparticle spacing of about $30 \mathrm{~nm}$ for maximum local SERS enhancement in the case of $1 \mathrm{D}$ Au nanodisk arrays. Spacing below $30 \mathrm{~nm}$ does not significantly affect the SERS enhancement. They suggested that for very small interparticle spacings, excitation of higher multipoles, in which the field enhancements are smaller than that of dipole mode, can induce the decrease in SERS enhancement. Such nonzero optimum interparticle spacing may also be an important fact to the high SERS intensity and its monotonous dependence on the molecule packing densities we observed.

The confinement of the self-assembled SBS template also enables a very high stability for the Ag nanoparticle arrays. Our previous work showed that the SBS-templated onedimensional Ag nanoparticle arrays have a much enhanced thermal stability as compared with the nanoparticle assemblies on the surfaces of covalent amorphous solids or even on the disordered SBS films. They were not influenced by long time thermal annealing at a temperature as high as $560 \mathrm{~K}$ [28]. We also evaluated the stability of the Ag nanoparticle substrates for SERS applications. The sample was stored in dry atmospheric environment for several weeks and reexamined. No significant changes on the SERS response and sensitivity were observed. From TEM observation, we confirmed that the morphology of the Ag nanoparticles deposited on SBS template surface did not change over time, even after immersion with BPE methanol solution. Although reusability cannot be expected for the $1 \mathrm{D}$ Ag nanoparticle array substrates in their current form, mass production of such SERS substrates is easy to be realized at significant low cost. This simple and straightforward method offers considerable potential for the fabrication of practical sensor chips based on SERS.

\section{Conclusions}

As a conclusion, an optimized SERS substrate has been demonstrated by fabricating densely packed one-dimensional silver nanoparticle arrays with a self-assembled block copolymer template. High SERS activity, stability, and reproducibility have been achieved due to the well-controlled patterning geometry of the nanoparticle arrays. The dependence of the average Raman enhancement factors and the SERS intensities on the packing density of the analyte molecules have been obtained. For a range of very low analyte density with a molecular coverage from $0.2 \%$ to $6 \%$ on 
the substrate surface; the SERS signal of the analytes exhibits a linear dependence on the molecule packing density on a logarithmic scale, with a slope of about 1.25 . Such onedimensional silver nanoparticle array-based SERS substrates are promising for quantitative trace level detection and rapid analysis of molecules.

\section{Conflict of Interests}

The authors state that none of the authors of this paper have a direct financial relation with the commercial identities mentioned in this paper that might lead to conflict of interests for them.

\section{Acknowledgments}

The authors thank the financial support from the National Basic Research Program of China (973 Program, contract no. 2009CB930501), NSFC (Grant nos. 51171077 and 10974092), as well as the Analysis and Measurement Foundation of Nanjing University.

\section{References}

[1] M. Fleischmann, P. J. Hendra, and A. J. McQuillan, "Raman spectra of pyridine adsorbed at a silver electrode," Chemical Physics Letters, vol. 26, no. 2, pp. 163-166, 1974.

[2] D. L. Jeanmaire and R. P. Van Duyne, "Surface Raman spectroelectrochemistry: part I. heterocyclic, aromatic, and aliphatic amines adsorbed on the anodized silver electrode," Journal of Electroanalytical Chemistry, vol. 84, no. 1, pp. 1-20, 1977.

[3] M. G. Albrecht and J. A. Creighton, "Anomalously intense Raman spectra of pyridine at a silver electrode," Journal of the American Chemical Society, vol. 99, no. 15, pp. 5215-5217, 1977.

[4] M. Moskovits, "Surface roughness and the enhanced intensity of Raman scattering by molecules adsorbed on metals," The Journal of Chemical Physics, vol. 69, no. 9, pp. 4159-4161, 1978.

[5] M. Moskovits, "Surface-enhanced Raman spectroscopy: a brief perspective," Topics in Applied Physics, vol. 103, pp. 1-17, 2006.

[6] H. Ko, S. Singamaneni, and V. V. Tsukruk, "Nanostructured surfaces and assemblies as SERS media," Small, vol. 4, no. 10, pp. 1576-1599, 2008.

[7] M. Geissler and Y. Xia, "Patterning: principles and some new developments," Advanced Materials, vol. 16, no. 15, pp. 12491269, 2004.

[8] Y. Fang, N.-H. Seong, and D. D. Dlott, "Measurement of the distribution of site enhancements in surface-enhanced Raman scattering," Science, vol. 321, no. 5887, pp. 388-391, 2008.

[9] D. A. Genov, A. K. Sarychev, V. M. Shalaev, and A. Wei, "Resonant field enhancements from metal nanoparticle arrays," Nano Letters, vol. 4, no. 1, pp. 153-158, 2004.

[10] W. A. Lopes and H. M. Jaeger, "Hierarchical self-assembly of metal nanostructures on diblock copolymer scaffolds," Nature, vol. 414, no. 6865, pp. 735-738, 2001.

[11] Z. Shi, M. Han, F. Song, J. Zhou, J. Wan, and G. Wang, "Hierarchical self-assembly of silver nanocluster arrays on triblock copolymer templates," Journal of Physical Chemistry B, vol. 110, no. 37, pp. 18154-18157, 2006.
[12] S. B. Darling, "Directing the self-assembly of block copolymers," Progress in Polymer Science, vol. 32, no. 10, pp. 1152-1204, 2007.

[13] Q. Li, J. He, E. Glogowski et al., "Responsive assemblies: gold nanoparticles with mixed ligands in microphase separated block copolymers," Advanced Materials, vol. 20, no. 8, pp. 14621466,2008

[14] Y. Wang, M. Becker, L. Wang et al., "Nanostructured gold films for SERS by block copolymer-templated galvanic displacement reactions," Nano Letters, vol. 9, no. 6, pp. 2384-2389, 2009.

[15] J. Lu, D. Chamberlin, D. A. Rider, M. Liu, I. Manners, and T. P. Russell, "Using a ferrocenylsilane-based block copolymer as a template to produce nanotextured Ag surfaces: uniformly enhanced surface enhanced Raman scattering active substrates," Nanotechnology, vol. 17, no. 23, pp. 5792-5797, 2006.

[16] C. H. Xu, B. Xie, Y. J. Liu, L. B. He, and M. Han, “Optimizing surface-enhanced Raman scattering by template guided assembling of closely spaced silver nanocluster arrays," European Physical Journal D, vol. 52, no. 1-3, pp. 111-114, 2009.

[17] S. Hadano, H. Handa, K. Nagai, T. Iyoda, J. Li, and S. Watanabe, "Surface-enhanced Raman scattering (SERS) effect of hexagonally arranged gold nanoparticle array with 29-nm particles and 23-nm gaps using liquid-crystalline block-copolymer template," Chemistry Letters, vol. 42, no. 1, pp. 71-73, 2013.

[18] W. J. Cho, Y. Kim, and J. K. Kim, "Ultrahigh-density array of silver nanoclusters for SERS substrate with high sensitivity and excellent reproducibility," ACS Nano, vol. 6, no. 1, pp. 249-255, 2012.

[19] Y. Liu, Y. C. Gong, L. B. He et al., "Formation of periodic nanoring arrays on self-assembled PS-b-PMMA film under rapid solvent-annealing," Nanoscale, vol. 2, no. 10, pp. 2065-2068, 2010.

[20] H. Haberland, M. Mal, M. Mosele, Y. Qiang, Th. Reiners, and Y. Thurner, "Filling of micro-sized contact holes with copper by energetic cluster impact (ECI)," Journal of Vacuum Science \& Technology A, vol. 12, no. 5, pp. 2920-2925, 1994.

[21] M. Han, C. H. Xu, D. Zhu et al., "Controllable synthesis of twodimensional metal nanoparticle arrays with oriented size and number density gradients," Advanced Materials, vol. 19, no. 19, pp. 2979-2983, 2007.

[22] T. L. Morkved, P. Wiltzius, H. M. Jaeger, D. G. Grier, and T. A. Witten, "Mesoscopic self-assembly of gold islands on diblockcopolymer films," Applied Physics Letters, vol. 64, no. 4, pp. 422424, 1994.

[23] R. L. Birke, C. Shi, W. Zhang, and J. R. Lombardi, "A time-resolved SERS study of the adsorption and electrochemical reduction of 4-pyridinecarboxaldehyde and 4-(hydroxymethyl) pyridine," Journal of Physical Chemistry B, vol. 102, no. 41, pp. 7983-7996, 1998.

[24] N. Félidj, J. Aubard, G. Lévi et al., “Controlling the optical response of regular arrays of gold particles for surface-enhanced Raman scattering," Physical Review B, vol. 65, no. 7, Article ID 075419, 9 pages, 2002.

[25] S. Zou and G. C. Schatz, "Silver nanoparticle array structures that produce giant enhancements in electromagnetic fields," Chemical Physics Letters, vol. 403, no. 1-3, pp. 62-67, 2004.

[26] L. Qin, S. Zou, C. Xue, A. Atkinson, G. C. Schatz, and C. A. Mirkin, "Designing, fabricating, and imaging Raman hot spots," Proceedings of the National Academy of Sciences of the United States of America, vol. 103, no. 36, pp. 13300-13303, 2006. 
[27] L. Qin, M. J. Banholzer, J. E. Millstone, and C. A. Mirkin, "Nanodisk codes," Nano Letters, vol. 7, no. 12, pp. 3849-3853, 2007.

[28] C. H. Xu, X. Chen, Y. J. Liu et al., "Enhanced thermal stability of monodispersed silver cluster arrays assembled on block copolymer scaffolds," Nanotechnology, vol. 21, no. 19, Article ID 195304, 2010 . 

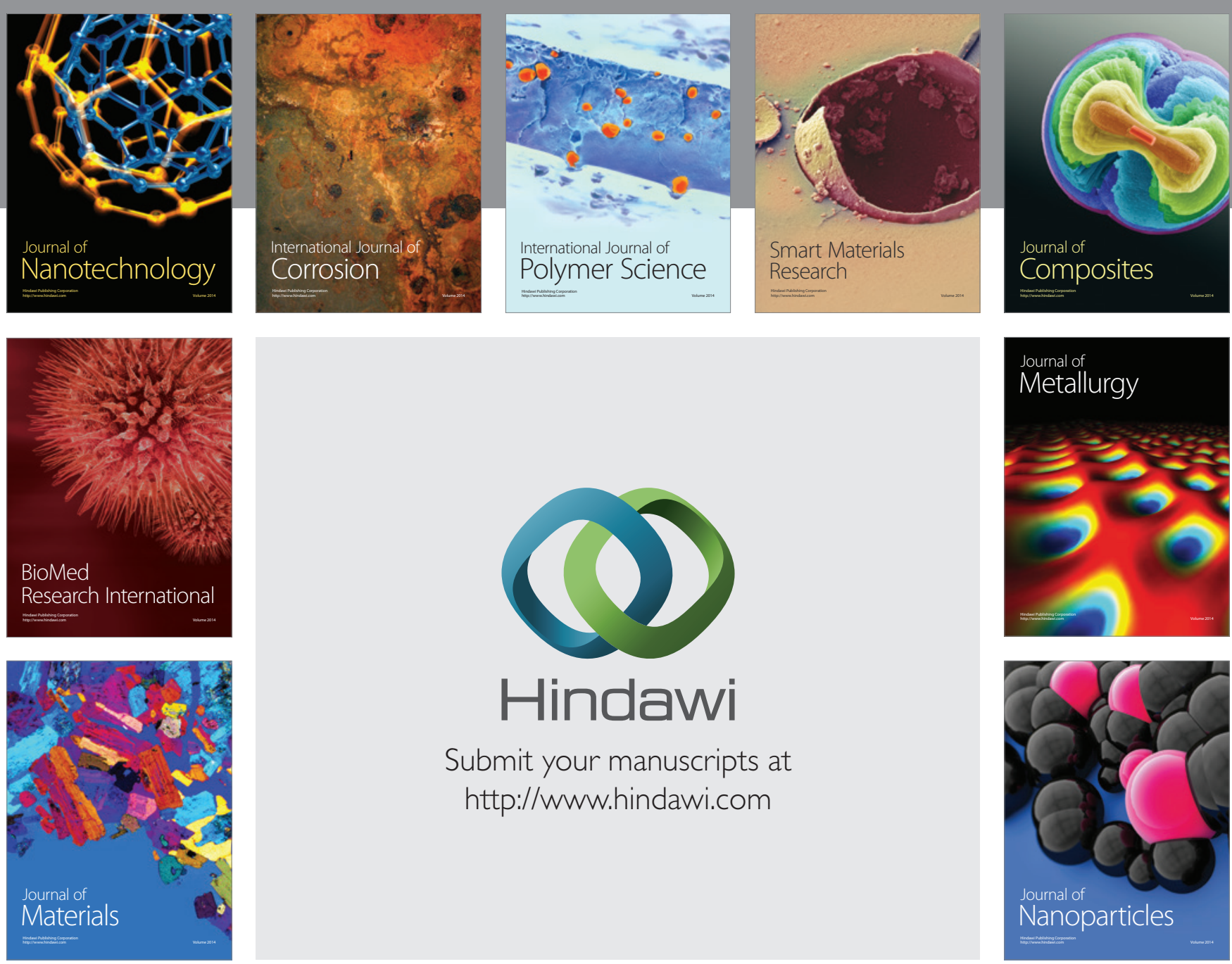

Submit your manuscripts at http://www.hindawi.com
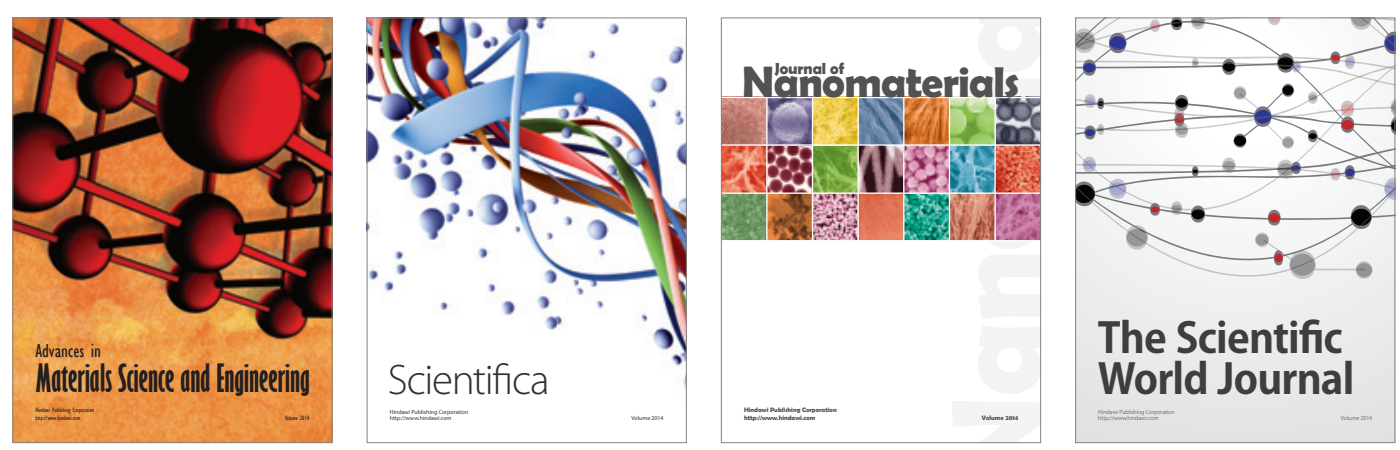

\section{The Scientific World Journal}
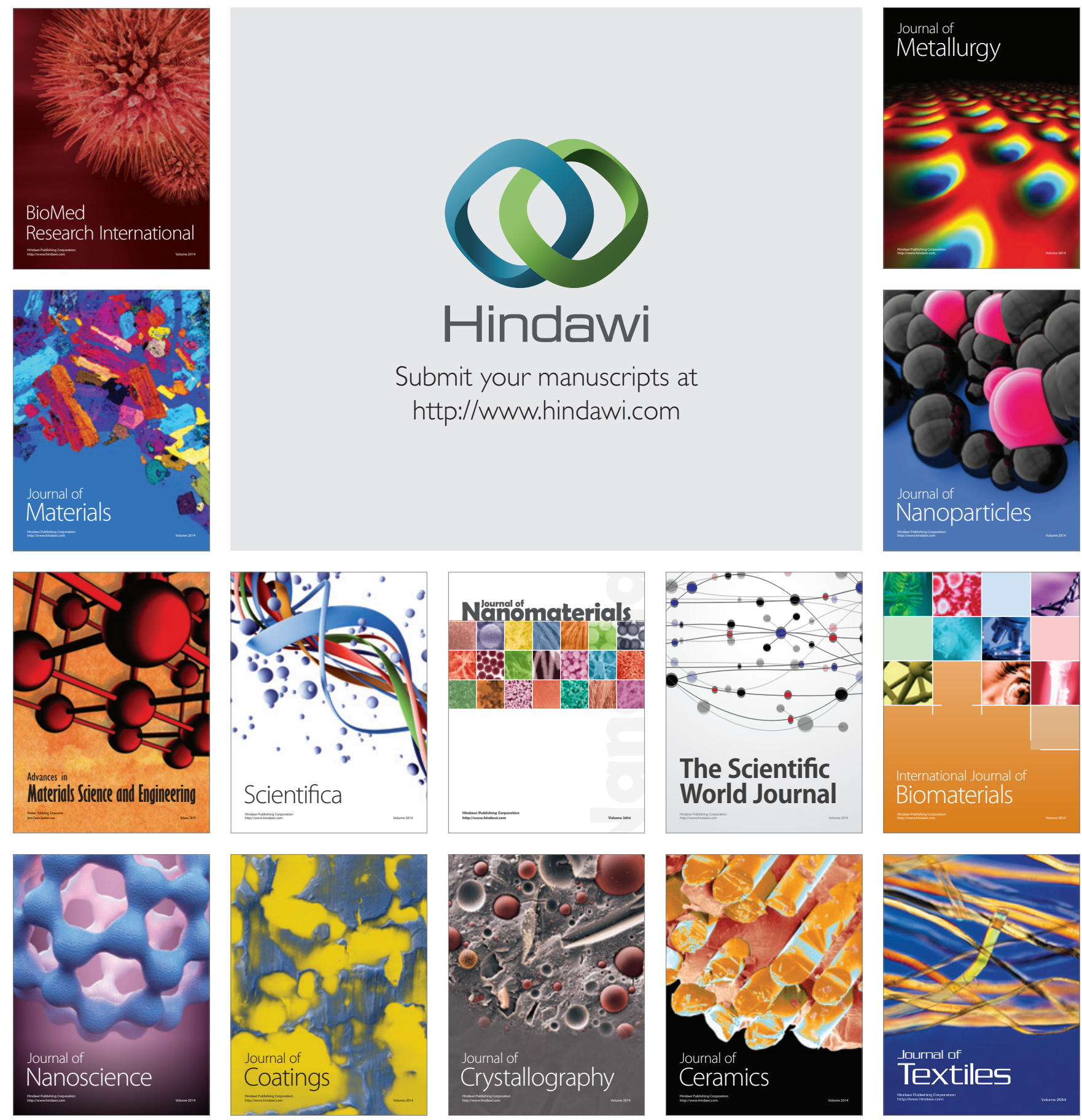